\title{
Ueber Psyllawachs, Psyllostearylalkohol und Psyllostearylsäure (Psyllaalkohol, Psyllasäure).
}

\author{
III. Mittheilung.
}

Von

Erust Edw. Sundwik.

(Aus dem physiologisch-chemischen Institut zu Helsingfors.)

(Der Redaction zugegangen am 30. März 1901.)

Ich habe in 3 Publicationen (diese Zeitschrift, Bde. XVII und $\mathrm{XXV}$ ) die Untersuchungen veröffentlicht, welche ich über das von Psylla Alni, einer auf Alnus incana lebenden Blattlaus, erzeugte Wachs angestellt habe. Da ich keine Säure bei der Spaltung wahrnehmen konnte, habe ich mich für berechtigt gehalten, die Substanz als einen Aether zu charakterisiren. Nunmehr ist es mir gelungen, auch eine Säure darzustellen und die Wachssubstanz als einen Ester von der Zusammensetzung $\mathrm{C}_{33} \mathrm{H}_{67} \cdot \mathrm{O} \cdot \mathrm{C}_{33} \mathrm{H}_{65} \mathrm{O}$ festzustellen. Die eigenartigen Eigenschaften der sehr schwachen Säure, die Unlöslichkeit auch ihrer Alkalisalze in Wasser und Alkohol neben anderen Umständen, mögen als entschuldigende Momente dienen, um so mehr, als ich stets mit nur geringen Mengen arbeiten konnte.

Das Psyllawachs wird, wie ich gezeigt habe, am besten durch halbstündiges Erhitzen im Oelbade bei $210-220^{\circ} \mathrm{C}$. mit Bromwasserstoffsäure von 1,49 specifischen Gewichts verseift. Beim Oeffnen des zugeschmolzenen Rohres nimmt man keinen Druck wahr. Die grobe, krystallinische Masse wird gepulvert, mit Wasser völlig von Säure befreit und nach dem Trocknen mit Chloroform im Extractionsapparate gelöst. Nur sehr geringe Spuren einer kohligen Masse bleiben ungelöst. Nach Verdunstung des Lösungsmittels bleibt der Stoff völlig farblos zurück. 
Die Scheidung der Bestandtheile konnte ich nach zwei Methoden bewirken. Erstens durch Zusatz von etwas starker Natronlauge zur alkoholischen Lösung, wobei sowohl Säure als Alkohol ausgeschieden wurden, jene als Natriumsalz. Ein Zusatz von Wasser begünstigt die Ausscheidung. Die Masse wird abfiltrirt, völlig ausgewaschen, getrocknet und mit Chloroform oder besser Aether oder Benzol im Extractionsapparat ausgezogen. Der Rückstand wird nach dem Trocknen in mit etwas Eisessig versetztem Alkohol auskrystallisirt.

Zweitens habe ich das Verseifungsprodukt in heissem Alkohol gelöst, mit Baryt und Chlorbaryum gefällt, ausgewaschen und weiterhin wie oben behandelt. Nur habe ich vor der Extraction den Baryt mit verdünnter, heisser Chlorwasserstoffsäure entfernt.

Aus Benzol, Petroleumäther, Chloroform und anderen Lösungsmitteln krystallisirt der Alkohol in seideglänzenden Schuppen, die Säure in viereckigen, rautenförmigen, dünnen Blättern, mit Winkeln von je $74^{\circ}$ und $106^{\circ}$. Es waren eben diese Krystalle, die ich als die dem Alkohol zugehörigen betrachtete. Es lag nahe, anzunehmen, dass die Krystalle, von denen ich stets nur wenig bekam, den besonders reinen Alkohol darstellten. Durch meine Arbeitsmethode ging früher der grösste Theil der Säure verloren.

Ich gehe nun zur Ermittelung der Zusammensetzung des Wachses, der Säure und des Alkohols über.

\section{Das Psyllawachs.}

Durch meine früher angeführten Elementaranalysen habe ich darthun können, dass die Formel desselben $\mathrm{C}_{33} \mathrm{H}_{66} \mathrm{O}$ oder ein Multiplum sein muss. Ich habe später mehrere Molekularbestimmungen gemacht, welche im Mittel 984 gaben; unter den Bestimmungen gab eine als Molekulargewicht die Zahl 954,6. Später habe ich noch eine solche Bestimmung gemacht mit folgendem Resultat:

$1,13 \mathrm{~g}$ in $50 \mathrm{ccm} . \mathrm{C}_{6} \mathrm{H}_{6}(=44 \mathrm{~g})$ entspricht $2,5 \%$ an Stoff. $\mathrm{t}^{0}-\mathrm{t}^{\prime 0}=0,068$, also Molekulargewicht gleich 981,6.

$\mathrm{C}_{33} \mathrm{H}_{66} \mathrm{O}$ entspricht dem Molekulargewicht 478. Die Formel muss also verdoppelt und $\mathrm{C}_{66} \mathrm{H}_{132} \mathrm{O}_{2}$ geschrieben werden, ent- 
sprechend dem Gewicht 956. Ein so geringer Fehler, wie hier vorhanden, kommt nicht in Betracht, da die Möglichkeit einer genauen Bestimmung bekanntlich mit der Grösse des Moleküls abnimmt.

Um zu erfahren, ob wirklich das Wachs in zwei fast ebenso grosse Moleküle zerfällt, habe ich zwei verschiedene Wege eingeschlagen:

a) $1 \mathfrak{g}$ des völlig verseiften und vor dem Wägen getrockneten Wachses wurde in Alkohol gelöst, mit Baryt gefällt, mit Wasser ausgewaschen und getrocknet. Dem getrockneten Produkt wurde der neue Alkohol mit Benzol entzogen, getrocknet und gewogen. Der ungelöste Rückstand wurde mit heisser, verdünnter Salzsäure behandelt, ausgewaschen, getrocknet und in derselben Weise extrahirt. Die Säure wurde als trocken gewogen.

Ich erhielt: Alkohol 0,52 g, Säure 0,57 g, zusammen 1,09 g. Die Alkoholmenge bezw. Säuremenge sollte sich wie 0,54:0,57 verhalten; die Zahlen sind also hinreichend genau.

b) $0,390 \mathrm{~g}$ der mit $\mathrm{HBr}$ verseiften Substanz, wohl getrocknet, wurde in $50 \mathrm{ccm}$. Benzol (= $=44 \mathrm{~g})$ gelöst. Lösung also $=0,886 \%$. $\mathrm{t}^{10}-\mathrm{t}^{0}$ $=0,049$; hieraus berechnet sich das Molekulargewicht zu 482 als Mittelgehalt von 1 Molekül Säure und 1 Molekül Alkohol, anstatt 487. Die Annahme, dass die beiden Moleküle fast gleich seien, ist auch hiermit bewiesen. Es gilt nunmehr, nur noch die Säure und den Alkohol zu analysiren bezw. deren Molekulargewicht zu bestimmen.

\section{Die Psyllostearylsäure (Psyllasäure).}

Die vorher genannte, aus Essigsäure enthaltendem Aethylalkohol auskrystallisirte Säure wurde analysirt und zwar mit folgendem Resultat:

$$
\begin{aligned}
& 0,182 \mathrm{~g} \text { gaben } 0,5342 \mathrm{~g} \mathrm{CO}_{\mathbf{g}}=0,1457 \mathrm{~g} \mathrm{C}=80,05 \% \text {, } \\
& \text { ebenso: } 0,2252 \mathrm{~g} \mathrm{H}_{2} \mathrm{O}=0,02502 \mathrm{~g} \mathrm{H}=13,75 \% \text {. } \\
& \mathrm{C}_{38} \mathrm{H}_{68} \mathrm{O}_{2}=\mathrm{C}_{98} \mathrm{H}_{65} \cdot \mathrm{COOH} \text { erfordert 80,16\% Cl und } 13,36 \% \mathrm{H} \text {. }
\end{aligned}
$$
Es war also die reine Säure vorhanden.

Ich habe, um sicher zu sein, auch noch das Molekulargewicht bestimmt:

$0,2575 \mathrm{~g}$ in $40 \mathrm{ccm} . \mathrm{C}_{6} \mathrm{H}_{6}$ gelöst (specifisches Gewicht $=0,882$ ), also $=35,28 \mathrm{~g} \mathrm{C}_{6} \mathrm{H}_{6}$. Die Lösung enthielt also $0,726 \%$ und das Molekulargewicht berechnet sich zu 497 (statt 494).

Die Zusammensetzung $\mathrm{C}_{33} \mathrm{H}_{66} \mathrm{O}_{2}$ zeigt, dass es nur eine einbasische Säure sein kann. 


\section{Der Psyllostearylalkohol (Psyllaalkohol).}

Aus dem Vorhergehenden ist ersichtlich, dass dem Alkohol nur die Zusammensetzung $\mathrm{C}_{33} \mathrm{H}_{67} \cdot \mathrm{OH}$ zukommen kann, in Anbetracht nicht nur der Spaltungsprodukte, sondern auch der Zusammensetzung des ursprünglichen Körpers. Die Spaltung kann nur in folgendem Sinne verlaufen:

$$
\mathrm{C}_{66} \mathrm{H}_{132} \mathrm{O}_{2}+\mathrm{H}_{2} \mathrm{O}=\underbrace{\mathrm{C}_{38} \mathrm{H}_{67} \cdot \mathrm{OH}}_{\text {Alkohol. }}+\underbrace{\mathrm{C}_{38} \mathrm{H}_{68} \mathrm{O}_{2}}_{\text {Säure. }} .
$$

Wie leicht einzusehen ist, ist auch die Möglichkeit einer zwei- oder mehrwerthigen Säure oder Alkohol ausgeschlossen. Ich habe dennoch eine Molekulargewichtsbestimmung vorgenommen:

$0,24 \mathrm{~g}$ des Alkohols wurde in $50 \mathrm{ccm} . \mathrm{C}_{6} \mathrm{H}_{6}(=0,88$ specifisches Gewicht), also $44 \mathrm{~g}$, gelöst. Die Lösung enthielt $0,5425 \%$ Stoff.

$\mathrm{t}^{0}-\mathrm{t}^{\prime 0}=0,029$ und das Molekulargewicht berechnet sich zu 496 anstatt 480 .

Hiermit dürfte also bewiesen sein, dass das Psyllawachs eine neutrale Verbindung eines einwerthigen Alkohols $\mathrm{C}_{33} \mathrm{H}_{67} \cdot \mathrm{OH}$ mit einer einbasischen Säure $\mathrm{C}_{32} \mathrm{H}_{65} \cdot \mathrm{COOH}$ ist. Die Eigenschaften des Wachses wie auch des Alkohols sind unverrückt dieselben, die ich in meinen früheren Publicationen ihnen zugetheilt habe.

Um noch sicherer zu sein, habe ich den Benzoesäureester des Alkohols dargestellt und analysirt.

Der Alkohol wurde mit der berechneten Menge Benzoesäureanhydrid im offenen Gefäss im Oelbade bis zu 150-1650 erhitzt, erkalten gelassen und mehrmals aus Benzol und Petroläther umkrystallisirt. Der bei $68-69^{\circ} \mathrm{schmelzende} \mathrm{Ester} \mathrm{gab}$ bei der Analyse folgende Zahlen:

$$
\begin{array}{r}
0,2440 \mathrm{~g} \text { gaben } 0,6915 \mathrm{~g} \mathrm{CO}_{2}=0,1886 \mathrm{~g} \mathrm{C}=77,29 \%, \\
\text { ebenso: } 0,2207 \mathrm{~g} \mathrm{H}_{2} \mathrm{O}=0,03008 \mathrm{~g} \mathrm{H}=12,33 \% .
\end{array}
$$

Die Formel $\mathrm{C}_{33} \mathrm{H}_{67} \cdot \mathrm{O} \cdot \mathrm{C}_{7} \mathrm{H}_{5} \mathrm{O}$ erfordert $77,42 \% \mathrm{C}$ und $12,26 \% \mathrm{H}$.

Ich lasse hier noch eine Tabelle über das Verhalten der oben behandelten Stoffe in physikalischer Hinsicht folgen: 


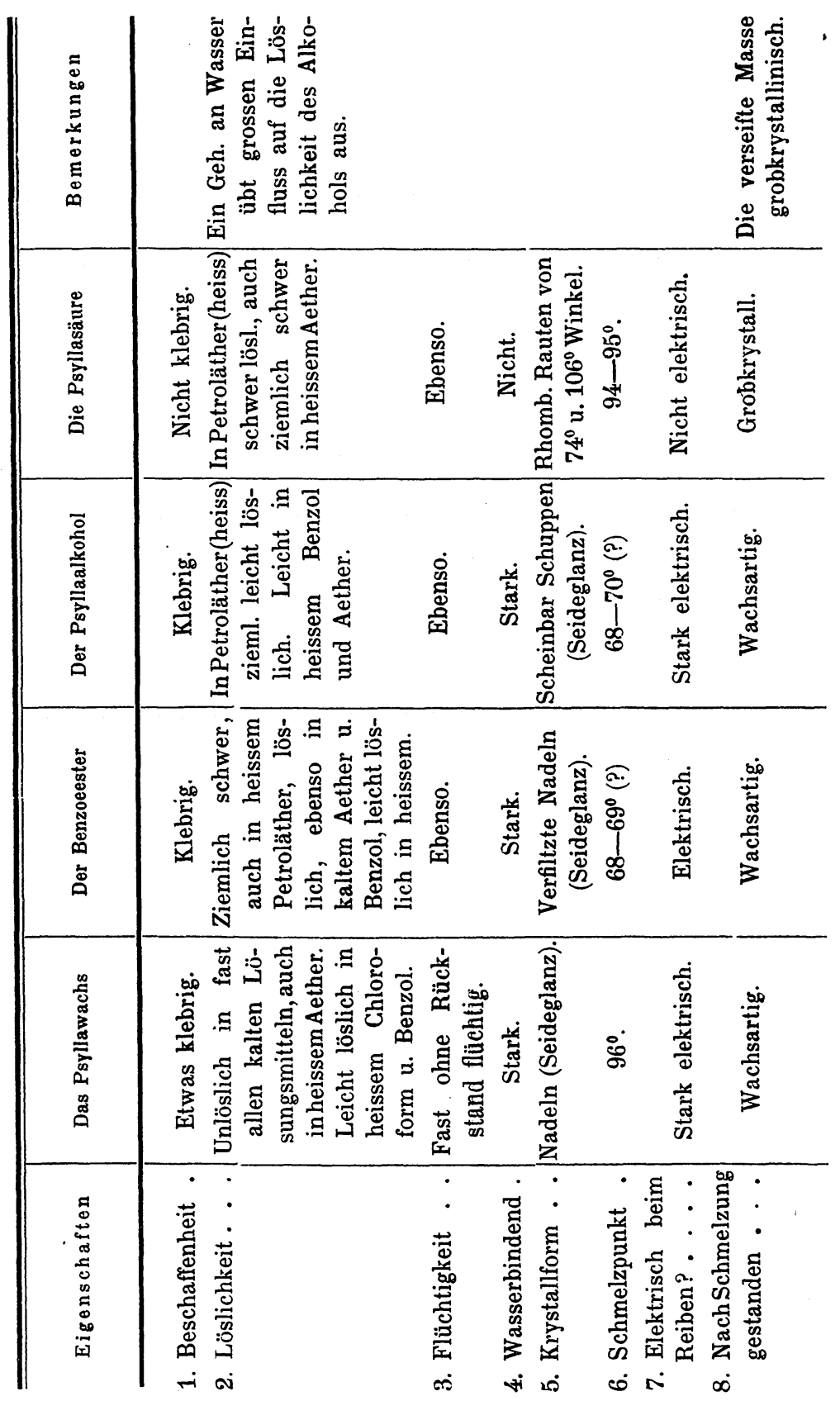


Die schwachen negativen Eigenschaften der Säure, wie auch der Umstand, dass die Salze unlöslich und amorph sind, machen es unmöglich, Analysen von Salzen zu erhalten, obgleich die schön krystallisirende Säure unschwer zu erhalten ist. Die Salze ziehen sich in das Filterpapier hinein und sind dann nach dem Trocknen nicht davon loszumachen. Auch das Silbersalz verhält sich ebenso, obgleich es ungewöhnlich beständig ist. So verfärbt es sich z. B. erst nach längerer Beleuchtung.

Ueber die Schmelzpunkte des Benzoesäureesters und des Alkohols bin ich nicht völlig sicher, da ja bekanntlich auch sehr geringe Beimischungen bedeutende Erniedrigungen zur Folge haben. Nach Beschaffung von etwas mehr Material beabsichtige ich eine neue Bestimmung zu machen.

Helsingfors, Physiologisch-chemisches Institut. 